\section{Prevalência e fatores associados à infecção pelo Mycobacterium tuberculosis entre agentes comunitários de saúde no Brasil, usando-se a prova tuberculínica}

\author{
Prevalence of infection with Mycobacterium \\ tuberculosis and associated factors in community \\ health workers in Brazil based on the \\ tuberculin skin test
}

\author{
Prevalencia y factores asociados a la infección por \\ Mycobacterium tuberculosis, usando la prueba \\ tuberculina entre los agentes comunitarios de \\ salud en Brasil
}

\author{
${ }^{1}$ Centro de Ciências da \\ Saúde, Universidade Federal \\ do Espírito Santo, Vitória, \\ Brasil. \\ 2 Departamento de Vigilância \\ Ambiental e Epidemiológica, \\ Secretaria Municipal de \\ Saúde de Manaus, Manaus, \\ Brasil. \\ Correspondência \\ E. L. N. Maciel \\ Departamento de \\ Enfermagem, Centro \\ de Ciências da Saúde, \\ Universidade Federal do \\ Espírito Santo. \\ Av. Marechal Campos 1468, \\ Vitória, ES 29040-091, Brasil. \\ ethel.maciel@gmail.com
}

\begin{abstract}
This article aimed to determine the prevalence of latent Mycobacterium tuberculosis infection and associated factors in community health workers (CHW) in Brazil, using two cutoff points for the tuberculin skin test $(5 \mathrm{~mm}$ and $10 \mathrm{~mm}$ ). This was a cross-sectional study with data on gender, age, BCG scar, working in a primary care unit (PCU), having a household contact with tuberculosis (TB), alcohol consumption, previous tuberculin skin test (TST), smoking, time on the job as CHW, and comorbidities. Logistic regression was used to estimate odds ratios (OR) and to control for confounding. TST was applied and read after 48-72 hours. Prevalence rates were $57.88 \%$ and $37.3 \%$, respectively, for the $5 \mathrm{~mm}$ and $10 \mathrm{~mm}$ TST cutoffs. Variables that remained associated with positive TST using the 10mm cutoff were working in a primary care unit that had implemented a Tuberculosis Control Program (TCP) and history of household contact with TB. Variables associated with the $5 \mathrm{~mm}$ cutoff were working in a primary care unit with a TCP. Necessary actions at the local level include awareness-raising and strengthening of continuing education.
\end{abstract}

Tuberculin Test; Tuberculosis; Mycobacterium tuberculosis; Community Health Workers
Wesley Pereira Rogerio 1

Thiago Nascimento do Prado 1

Fernanda Mattos de Souza 1

Jair dos Santos Pinheiro 2

Patrícia Marques Rodrigues 1

Amanda Pissinate do Nascimento Sant'anna 1

Kássia Gomes de Jesus 1

Crispim Cerutti Junior 1

Rita de Cássia Duarte Lima 1

Ethel Leonor Noia Maciel 1

\section{Resumo}

Este artigo tem por objetivo determinar a prevalência e os fatores associados à infecção latente pelo Mycobacterium tuberculosis entre agentes comunitários de saúde (ACS), usando dois pontos de corte da prova tuberculínica $5 \mathrm{~mm}$ e $10 \mathrm{~mm}$. Trata-se de estudo transversal, com dados coletados sobre sexo, idade, cicatriz de BCG, prova tuberculinica (PT) anterior, tempo que trabalha na profissão de ACS, atuar em unidade básica de saúde (UBS), ter tido contato intradomiciliar com tuberculose (TB), usar álcool, ser fumante e apresentar comorbidades. Para controle de variáveis de confusão e estimativa da medida de efeito (OR), foi empregada a regressão logística. Aplicou-se PT, com leitura após 48-72 horas. As prevalências foram de 57,88\% e 37,3\%, respectivamente, para $5 \mathrm{~mm}$ e $10 \mathrm{~mm}$. Manteve-se associada à positividade para o ponto de corte de $10 \mathrm{~mm}$ a condição de trabalhar em UBS com Programa de Controle de Tuberculose (PCT) implementado e já ter tido contato intradomiciliar com TB. Já para o ponto de corte de $5 \mathrm{~mm}$, trabalhar em UBS com PCT e implementado. São necessárias ações de conscientização nos municípios e fortalecimento das ações de educação permanente sobre a temática.

Teste Tuberculínico; Tuberculose;

Mycobacterium tuberculosis; Agentes

Comunitários de Saúde 


\section{Introdução}

A tuberculose (TB) ainda é um sério problema de saúde pública e demonstra relação direta com a pobreza. Está associada a processos históricos de exclusão social e marginalização de parte da população, submetida a más condições de vida, como moradia precária, desnutrição e dificuldade de acesso aos serviços e bens públicos. Assim, por ser uma das mais importantes causas de morbidade e mortalidade em vários países atualmente $1,2,3$, a TB configura-se como uma das principais doenças a serem enfrentadas no Brasil e no mundo 1 . Durante o curso da doença ativa, os pacientes infectam, em média, dez outros indivíduos, perpetuando a cadeia de transmissão da TB na comunidade 2 .

A Organização Mundial da Saúde (OMS) aponta 22 países que concentram cerca de $80 \%$ dos casos de TB. O Brasil ocupa a 16a posição em número absoluto de casos; ao ser considerado o coeficiente de incidência, o Brasil encontra-se na 22a posição entre esses países 4 . Estima-se que, em 2012, cerca de 8,6 milhões de pessoas tiveram TB no mundo; no entanto, observa-se redução do número de casos ao longo dos últimos anos. O número de mortes por TB no mundo também foi reduzido: estima-se que, em 1990, ocorreu 1,3 milhão de mortes por TB, ao passo que, em 2012, esse número foi de 940 mil, uma redução de $45 \% 4$.

No Brasil, em 2013, o coeficiente de incidência era de 35,4/100 mil habitantes. Destaca-se que esse coeficiente apresenta redução ao longo dos anos. Em 2003, o valor foi de 44,4/ 100 mil habitantes, uma redução de 20,4\% em comparação a 20131.

Do total de casos novos diagnosticados em 2013, 85,7\% apresentaram a forma clínica pulmonar; destes, 65,2\% eram bacilíferos. Quando não tratados adequadamente até a cura, esses casos são os principais responsáveis pela manutenção da cadeia de transmissão da doença. No ano de 2012, 70,6\% das ocorrências de TB pulmonar bacilífera tiveram cura, enquanto $10,5 \%$ abandonaram o tratamento 1 . A OMS recomenda a cura de pelo menos $85 \%$ dos casos de TB pulmonar bacilífera 4 .

Como forma de lidar com tal quadro, o Programa Nacional de Controle da Tuberculose (PNCT) incorpora, nesse enfrentamento, a capilaridade dos modelos assistenciais contidos tanto na Estratégia Saúde da Família (ESF), quanto no Programa de Agentes Comunitários de Saúde (PACS), na perspectiva de que essa parceria possa contribuir para a expansão das ações de controle da TB. A organização da ESF toma como base os territórios vivos onde as pessoas vivem e organi- zam a vida, constituindo-se em fio condutor para a organização desse modelo. Para Maciel et al. 5, o território é lugar de produção de relações, no qual se vão tecendo teias de afetos, encontros e desencontros, permeados por relações de poder, confiança, desconfiança, trabalho, adesões, boicotes e solicitações de serviços, além de equipamentos públicos diversos. Nesse sentido, a ESF privilegia a ênfase no trabalho em equipe, contando com a possibilidade de ampliar a detecção de casos, melhorar a adesão terapêutica e reduzir o abandono ao tratamento 6 .

Dentre os membros da equipe, os agentes comunitários de saúde (ACS) têm papel fundamental e aglutinador, ao se constituírem como elo e porta-voz das necessidades e demandas da comunidade em relação às possibilidades de ofertas dos serviços de saúde. Além de residirem na localidade em que atuam, mostram-se familiarizados com seus valores, costumes e linguagem, podendo, assim, produzir uma mescla entre o uso da tecnologia/conhecimento biomédico e as crenças locais. Agem, portanto, como tradutores, constroem pontes entre os serviços de saúde e a comunidade, identificando prontamente os problemas desta, facilitando o trabalho de prevenção de doenças e de promoção da saúde 7 .

Apesar de definitivamente incorporado ao controle da TB em diversas partes do mundo, em especial onde o tratamento diretamente observado apresenta alta cobertura, pouco se conhece das condições ocupacionais dos ACS no PNCT de nosso país 2 . No Brasil, foram publicados três artigos sobre infecção latente de tuberculose (ILTB) em ACS 8,9,10, tendo sido encontradas altas taxas de infecção entre aqueles que acompanharam pacientes com TB em suas rotinas de serviço. Esses agentes apresentaram risco de infecção maior do que os que não acompanharam pacientes com a doença. Contudo, os casos restringiram-se a somente um único cenário e com tamanho amostral pequeno.

Nesse contexto, a latência da ILTB consiste no período entre a entrada do Mycobacterium tuberculosis no organismo e o aparecimento da TB. A detecção de ILTB permite a instituição de tratamento medicamentoso para os indivíduos infectados que apresentam maior risco de progressão para doença ativa, sendo uma das estratégias de controle da TB conhecidas e recomendadas pela OMS 11 .

Considerando-se os grupos de risco para ILTB, os profissionais da área da saúde estão entre os mais vulneráveis, como demonstram várias publicações 12,13,14,15,16,17,18,19,20. Uma revisão sistemática encontrou que a incidência de ILTB, demonstrada por meio da conversão da prova tuberculínica (PT), pode chegar a $14 \%$ nesses 
profissionais, e o risco de evoluir para TB doença é de 5.361 casos / 100 mil profissionais de saúde a cada ano, em virtude da exposição nosocomial 21. Somado a isso, Roth et al. 22 , por meio de um estudo multicêntrico, realizado em quatro hospitais de diferentes regiões do Brasil, identificaram uma taxa de ILTB de cerca de $60 \%$ entre profissionais de saúde, sendo os principais fatores de risco para a contaminação: o local de trabalho, que é o hospital, o cargo de enfermeiro e a história de exposição à TB em ambiente hospitalar.

Desde o final do século XIX, a PT, realizada mediante o uso do derivado de proteína purificado (purified protein derivative - PPD), tem sido o método de escolha para detecção da ILTB. No Brasil, indivíduos com a infeção são detectados por meio da presença de uma PT positiva associada à exclusão de TB doença 23 . A classificação da PT mudou em 2010: atualmente, o ponto de corte sugestivo de infecção por TB em maiores de dois anos é $5 \mathrm{~mm}$, concluindo-se que o ponto de corte da PT de $5 \mathrm{~mm}$ de enduração pode ser utilizado mesmo em regiões com alta prevalência de TB ativa 23,24 .

Sendo assim, o presente estudo tem como objetivo estimar a prevalência e os fatores associados à infecção latente pelo $M$. tuberculosis entre ACS atuantes na rede básica de saúde de municípios prioritários para o controle de TB, como Cuiabá (Mato Grosso), Manaus (Amazonas), Salvador (Bahia) e Vitória (Espírito Santo). Além disso, tem o intuito de avaliar a redução do ponto de corte para $5 \mathrm{~mm}$, conforme proposto pela nova diretriz brasileira.

\section{Método}

\section{Tipo de estudo}

Estudo transversal conduzido entre 2011 e 2012, em quatro cidades brasileiras prioritárias para controle da TB, com alta incidência da doença ( $\geq 47 / 100$ mil habitantes), para o ano de 2012 . As cidades investigadas são as seguintes: Vitória (39,9/100 mil), Cuiabá (51,7/100 mil), Salvador (59,8/100 mil) e Manaus (71,2/100 mil) (Departamento de Informática do SUS. Tabnet. http:// tabnet.datasus.gov.br/cgi/tabcgi.exe?idb2010/ d0202.def, acessado em 08/Jul/2013). Vitória, capital do Espírito Santo, está situada na Região Sudeste do Brasil, com população de 327.801 habitantes; conta com cobertura de $90 \%$ de saúde da família e um sistema de informação sobre TB integrado ao laboratório de referência. O Município de Manaus, capital do Estado do Amazonas, possui população estimada em 1.688.524 habitantes, concentrando $50 \%$ da população do estado e $68 \%$ dos casos notificados de TB; conta com 46,8\% de ESF. Cuiabá, capital do Mato Grosso, está situada na região Centro-oeste do Brasil, com população de 551.098 habitantes. Salvador, capital da Bahia, localiza-se na região Nordeste, com população de 2.902.927 habitantes (Instituto Brasileiro de Geografia e Estatística. Censo Demográfico 2010. http://www.censo2010.ibge. gov.br, acessado em 08/Jul/2013).

\section{População de estudo}

A população do estudo foi composta por ACS, sendo $69(21,5 \%)$ em Manaus, 76 (23,6\%) em Salvador, 108 (33,6\%) em Vitória e 68 (21,1\%) em Cuiabá. Os critérios de inclusão foram os seguintes: ser ACS; atuar nas unidades básicas de saúde (UBS) selecionadas por sorteio nos municípios estabelecidos; aceitar participar voluntariamente do estudo; assinar um Termo de Consentimento Livre e Esclarecido (TCLE); ter sido submetido à aplicação e leitura da PT. Os critérios de exclusão envolveram estado conhecido de HIV, infecção pelo HIV com base no teste rápido, TB prévia ou em tratamento.

\section{Amostragem}

O tamanho da amostra foi calculado a fim de estimar a taxa de prevalência de infecção pelo $M$. tuberculosis em ACS, com nível de 95\% de precisão. A amostragem foi realizada em dois estágios, considerando-se a população de ACS em cada cenário e a heterogeneidade entre os sistemas de saúde. Primeiro, foi realizado um levantamento das taxas de incidência de TB de todas as formas no ano 2007, nas capitais brasileiras 25 . Nesta etapa, foram escolhidas as quatro capitais com maiores incidência da doença, representando as regiões geográficas brasileiras. Na segunda etapa, foram escolhidos os estabelecimentos de saúde nos quais seria efetuada a coleta de dados.

Inicialmente, fez-se a divisão dos serviços de saúde dentro das unidades sanitárias (UBS e unidade de saúde da família - USF). Em seguida, foi efetuado o cálculo do tamanho amostral em cada estabelecimento de saúde escolhido, por meio de uma amostra estratificada por partilha proporcional. Em cada um dos municípios de estudo, foi levantado o número de ACS dos serviços, tendo como fonte as Secretarias Municipais de Saúde (SMS), fazendo-se, então, a distribuição do percentual dos agentes em cada categoria sanitária. Uma vez que seriam entrevistados, ao todo, duzentos profissionais/trabalhadores de saúde em cada município, calculou-se o número total de ACS que deveriam ser entrevistados. Para o cálculo, utilizou-se a função sampsi do programa 
Stata versão 11.0 (StataCorp LP, College Station, Estados Unidos).

\section{Variáveis}

Os dados demográficos coletados por meio das entrevistas, levando-se em conta os fatores associados à PT positiva nos pontos de corte de $5 \mathrm{~mm}$ e 10mm (variáveis independentes), incluíram sexo, idade, presença de cicatriz de vacina Bacillus Calmette-Guérin (BCG), trabalhar apenas em unidades de cuidados primários de saúde, entrar em contato com um membro da família com TB, ingesta de álcool, PT anterior, ser fumante, quantidade de anos na profissão de ACS e comorbidade (variáveis dependentes).

\section{Prova tuberculínica}

Imediatamente após a entrevista padronizada, um teste de Mantoux (Statens Serum Institut de Copenhague, Copenhage, Dinamarca), contendo $0,1 \mathrm{~mL}$ de PPD RT23 (tuberculina PPD Evans $2 \mathrm{UT}$ ), foi administrado via intradérmica por enfermeiros treinados. O endurecimento foi medido após 48-72 horas da administração do PPD e interpretado pelos mesmos profissionais. Foram utilizados dois pontos de corte da prova tuberculínica: $\geq 5 \mathrm{~mm}$ e $\geq 10 \mathrm{~mm}^{3,23}$.

\section{Teste rápido para HIV}

Uma amostra de sangue foi coletada para o teste rápido de HIV (Rapid Check-HIV 1 \& 2. Núcleo de Doenças Infecciosas, Universidade Federal do Espírito Santo, Vitória, Brasil), como procedimento de triagem e critério de exclusão. A coleta foi realizada no laboratório de referência em cada capital (Laboratório Municipal de Cuiabá; Laboratório Municipal de Salvador; Laboratório de Imunologia do Núcleo de Doenças Infecciosas, Universidade Federal do Espírito Santo, em Vitória e, no Laboratório de Microbiologia da Fundação de Medicina Tropical Dr. Heitor Vieira Dourado em Manaus).

\section{Análise dos dados}

A prevalência de infecção por TB foi estimada pelo resultado da PT 3,23. Foram empregados os seguintes testes: qui-quadrado ( 2), para diferenças de proporção; teste exato de Fisher, quando apropriado; e teste t de Student, para diferenças de médias. Os dados foram analisados no programa estatístico Stata versão 11.0, utilizando-se p $\leq$ 0,05 como nível de significância. Para controle de variáveis de confusão e estimativa da medida de efeito (odds ratio - OR), empregou-se a regressão logística; dessa forma, as variáveis foram entrando no modelo segundo uma seleção gradual das variáveis (backward stepwise), de acordo com teste de razão de verossimilhança (likelihood ratio test). Para a permanência da variável no modelo, utilizou-se valor de $\mathrm{p}<0,1$; para avaliar a significância, empregou-se o teste de Wald 26; para variáveis categóricas ordinais, foi aplicado um teste de tendência linear. Como ponto de corte para significância em todas as provas estatísticas, foi usado um valor de p de 0,05 bicaudal.

\section{Aspectos éticos}

Este estudo foi previamente autorizado pelas secretarias municipais de saúde dos municípios envolvidos e obteve aprovação do Comitê de Ética em Pesquisa com Seres Humanos do Centro de Ciências da Saúde, Universidade Federal do Espírito Santo (UFES), sob o registro CEP 007/2010. Foi solicitado um TCLE a todos os sujeitos do estudo antes que qualquer procedimento relacionado a eles fosse executado. Os ACS com resultado do teste rápido para HIV positivo foram encaminhados para avaliação em um centro de referência.

\section{Resultados}

Entre 2011 e 2012, 322 ACS aceitaram participar do estudo; no entanto, dez (3,1\%) participantes foram excluídos por não voltarem para a leitura da PT; quatro $(1,2 \%)$ tiveram TB ativa ou estavam em tratamento de TB e um $(0,3 \%)$ foi positivo para infecção pelo HIV (teste rápido positivo), como ilustrado pelo fluxograma (Figura 1).

Conforme evidenciado na Tabela 1, a maioria da população estudada $(89,6 \%)$ compôs-se de indivíduos do sexo feminino; a idade média foi 41,05 anos (22-66 anos); 275 (87,3\%) tinham cicatriz de vacina BCG e o tempo médio de trabalho na atenção básica foi de 9,1 anos (IC95\%: 8,6-9,7). Quando os resultados da PT foram considerados separadamente, a prevalência global de ILTB entre os ACS, de acordo com pontos de corte de $5 \mathrm{~mm}$ e $10 \mathrm{~mm}$, foi de $57,88 \%$ (IC95\%: 52,0-63,0) e 37,30\% (IC95\%: 31,0-42,0), respectivamente. As variáveis grau de instrução, comorbidade e uso de alguma droga imunossupressora não se associaram ao desfecho. Já as variáveis ser fumante, em ambos os pontos de corte; já ter convivido ou não com alguém com TB, no ponto de corte de $10 \mathrm{~mm}$; e já ter realizado PT anteriormente, no ponto de corte de $10 \mathrm{~mm}$, mostraram-se associadas ao desfecho.

A Tabela 2 apresenta a distribuição das características ocupacionais relacionadas à bios- 
Fluxograma de participação no estudo e resultados da prova tuberculínica (PT) de agentes comunitários da saúde (ACS) da atenção básica. Brasil, 2013.

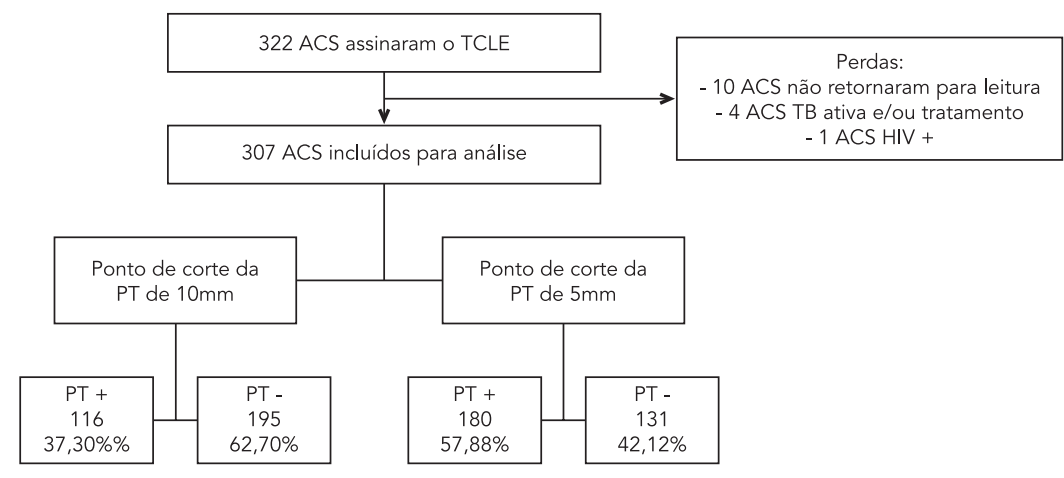

TCLE: Termo de Consentimento Livre e Esclarecido.

Nota: ponto de corte ao resultado da PT - PT+ $(\geq 10 \mathrm{~mm}$ e $\geq 5 \mathrm{~mm}$ de enduração) e PT- ( $<10 \mathrm{~mm}$ e $<5 \mathrm{~mm}$ de enduração).

segurança de ACS da atenção básica. Observa-se que, quanto aos dados ocupacionais, 122 (38\%) e $187(58,2 \%)$ dos agentes, nos pontos de corte de $10 \mathrm{~mm}$ e de $5 \mathrm{~mm}$, respectivamente, afirmaram trabalhar em UBS com PCT implantado. Essa variável não se mostrou associada à positividade para ambos os pontos de corte $(p=0,7)$. Em relação à variável frequência de equipamento de proteção individual (EPI) disponível, 231 (71,7\%) indivíduos responderam que o EPI às vezes se encontra disponível. Ainda, dentre os que responderam a esta opção, 91 (39,5\%) (ponto de corte de $10 \mathrm{~mm}$ ) e 132 (57,3\%) (ponto de corte de $5 \mathrm{~mm}$ ) apresentaram positividade à PT. Obtevese associação significante para a facilidade de acessibilidade ao EPI no ponto de corte de $10 \mathrm{~mm}$ da PT ( $\mathrm{p}=0,04)$.

Dentre os ACS que receberam capacitação sobre TB, para 134 (41,6\%), a ação se deu no último ano; 93 (28,8\%) relataram ter participado há um ano ou mais, referente ao período no qual a entrevista foi realizada. Não se obteve associação entre frequência de acesso ao conhecimento da TB e positividade à PT.

Empregou-se a regressão logística com o intuito de identificar os preditores da positividade do resultado da PT para os pontos de corte de $5 \mathrm{~mm}$ e $10 \mathrm{~mm}$. Das variáveis mensuradas, já ter tido contato intradomiciliar com alguém com TB permaneceu associada à positividade no modelo multivariado, para o ponto de corte de $10 \mathrm{~mm}$ da PT. Já para o ponto de corte de $5 \mathrm{~mm}$ da PT, a variável ter recebido treinamento sobre TB nos últimos dois anos manteve-se associada à positividade ao teste, conforme descrito na Tabela 3.

\section{Discussão}

Com base na nova proposta do Ministério da Saúde 27 a prevalência de $37,3 \%$ de infecção latente pelo $M$. tuberculosis, aumentada em $57,8 \%$, vem confirmar a hipótese de alta positividade à PT entre os ACS da atenção básica, que, na análise em questão, foi estimada para Cuiabá, Manaus, Salvador e Vitória. Estudos semelhantes envolvendo profissionais de saúde encontraram percentuais elevados de positividade à PT, variando de $26,7 \%$ a $69,5 \% 28,29$.

Permaneceram associadas à positividade no modelo multivariado para o ponto de corte da PT em $10 \mathrm{~mm}$ as variáveis trabalhar em unidade de saúde com PCT implementado e ter tido contato intradomiciliar com alguém com TB. Já para o ponto de corte da PT para $5 \mathrm{~mm}$, ter recebido treinamento sobre TB nos últimos dois anos manteve-se associada à positividade, enquanto ter recebido treinamento sobre TB nos últimos dois anos mostrou-se como fator de proteção.

Entende-se que os dados de prevalência de TB do presente estudo merecem cautela, pois, assim como a maioria daqueles publicados para a determinação do risco ocupacional, utilizou-se 
Tabela 1

Distribuição das características sociodemográficas e história clínica entre agentes comunitários de saúde (ACS) da atenção básica. Brasil, 2013.

\begin{tabular}{|c|c|c|c|c|c|c|c|c|}
\hline \multirow[t]{2}{*}{ Variáveis } & \multicolumn{4}{|c|}{ PT ponto de corte $\geq 10 \mathrm{~mm}$} & \multicolumn{4}{|c|}{ PT ponto de corte $\geq 5 \mathrm{~mm}$} \\
\hline & $\begin{array}{l}\mathrm{PT}+ \\
\mathrm{n}(\%)\end{array}$ & $\begin{array}{l}\text { PT- } \\
\text { n (\%) }\end{array}$ & $\begin{array}{l}\text { Razão de } \\
\text { prevalência }\end{array}$ & $\begin{array}{l}\text { Valor } \\
\text { de p }\end{array}$ & $\begin{array}{l}\text { PT+ } \\
\text { n (\%) }\end{array}$ & $\begin{array}{l}\text { PT- } \\
\text { n (\%) }\end{array}$ & $\begin{array}{l}\text { Razão de } \\
\text { prevalência }\end{array}$ & $\begin{array}{l}\text { Valor } \\
\text { de } p\end{array}$ \\
\hline \multicolumn{9}{|l|}{ Sexo } \\
\hline Masculino & $14(42,40)$ & $19(57,60)$ & 0,49 & 0,40 * & $19(57,60)$ & $14(42,40)$ & 0,73 & 0,90 * \\
\hline Feminino & $102(36,30)$ & $17(63,70)$ & & & $162(57,70)$ & $119(42,30)$ & & \\
\hline \multicolumn{9}{|l|}{ Idade (anos) } \\
\hline$<35$ & $93(62,42)$ & $104(63,03)$ & 0,33 & 1,00 & $95(57,58)$ & $70(42,42)$ & 0,83 & 0,88 \\
\hline$\geq 35$ & $56(37,58)$ & $61(36,97)$ & & & $87(58,39)$ & $62(41,61)$ & & \\
\hline \multicolumn{9}{|l|}{ Formação } \\
\hline Nível Fundamental & $2(40,00)$ & $3(60,00)$ & & & $2(40,00)$ & $3(60,00)$ & & \\
\hline Médio & $67(34,40)$ & $128(65,60)$ & 2,79 & 0,40 ** & $92(47,20)$ & $103(52,80)$ & 0,61 & 0,08 ** \\
\hline Técnico & $10(40,00)$ & $15(60,00)$ & & & $9(36,00)$ & $16(64,00)$ & & \\
\hline Superior & $39(43,80)$ & $50(56,20)$ & & & $29(32,60)$ & $60(67,40)$ & & \\
\hline \multicolumn{9}{|l|}{$\begin{array}{l}\text { Classificação dos municípios quanto à } \\
\text { prevalência de TB }\end{array}$} \\
\hline $\begin{array}{l}\text { Alta prevalência (Cuiabá, Manaus e } \\
\text { Salvador) }\end{array}$ & \multicolumn{7}{|c|}{ Salvador) } & 0,04 \\
\hline Média prevalência (Vitória) & $84(38,71)$ & $66(64,71)$ & & & $134(61,75)$ & $51(50,00)$ & & \\
\hline \multicolumn{9}{|l|}{ Possui alguma comorbidade ou faz uso de } \\
\hline \multicolumn{9}{|l|}{ alguma droga imunossupressora } \\
\hline Sim & $85(37,60)$ & $141(62,40)$ & 2,70 & 0,60 & $129(57,10)$ & $97(42,90)$ & 0,96 & 0,70 \\
\hline Não & $30(34,90)$ & $56(65,10)$ & & & $35(40,70)$ & $51(59,30)$ & & \\
\hline \multicolumn{9}{|l|}{ Fumante } \\
\hline Sim & $39(48,10)$ & $42(51,90)$ & 6,64 & 0,01 & $56(69,10)$ & $25(30,90)$ & 0,92 & 0,01 \\
\hline Não & $78(33,30)$ & $156(66,70)$ & & & $126(53,90)$ & $108(46,10)$ & & \\
\hline \multicolumn{9}{|l|}{ Etilista } \\
\hline Sim & $80(39,80)$ & $121(60,20)$ & 0,38 & 0,20 & $121(60,20)$ & $80(39,80)$ & 1,06 & 0,30 \\
\hline Não & $36(33,10)$ & $73(66,90)$ & & & $59(54,10)$ & $50(45,90)$ & & \\
\hline \multicolumn{9}{|l|}{ Apresenta cicatriz vicinal de BCG } \\
\hline Sim & $103(36,70)$ & $178(63,40)$ & 2,18 & 0,60 & $167(59,40)$ & $114(40,60)$ & 3,10 & 0,08 \\
\hline Não & $14(41,20)$ & $20(58,80)$ & & & $15(44,10)$ & $19(55,90)$ & & \\
\hline \multicolumn{9}{|l|}{ Já conviveu com alguém com TB? } \\
\hline Sim & $39(53,40)$ & $34(46,60)$ & 0,39 & 0,00 & $49(67,10)$ & $24(32,90)$ & 1,04 & 0,06 \\
\hline Não & $79(32,80)$ & $162(67,20)$ & & & $132(54,70)$ & $109(45,30)$ & & \\
\hline \multicolumn{9}{|l|}{ Já fez PT? } \\
\hline Sim & $36(47,40)$ & $40(52,60)$ & 1,55 & 0,03 & $50(65,70)$ & $26(34,30)$ & 0,70 & 0,07 \\
\hline Não & $79(33,70)$ & $155(66,30)$ & & & $127(54,30)$ & $107(45,70)$ & & \\
\hline
\end{tabular}

BCG: bacilo Calmete Guerin; PT: prova tuberculínica; TB: tuberculose.

Nota: ponto de corte para PT - PT+ $(\geq 10 \mathrm{~mm}$ e $\geq 5 \mathrm{~mm}$ de enduração) e PT- (< 10mm e $<5 \mathrm{~mm}$ de enduração).

* Teste de qui-quadrado de Pearson;

** Teste exato de Fisher. 
Tabela 2

Distribuição das características ocupacionais e relacionadas à biossegurança entre agentes comunitários de saúde (ACS) da atenção básica. Brasil, 2013.

\begin{tabular}{|c|c|c|c|c|c|c|c|c|}
\hline \multirow[t]{3}{*}{ Variáveis } & \multicolumn{4}{|c|}{ PT ponto de corte $\geq 10 \mathrm{~mm}$} & \multicolumn{4}{|c|}{ PT ponto de corte $\geq 5 \mathrm{~mm}$} \\
\hline & PT+ & PT- & $\begin{array}{l}\text { Razão de } \\
\text { prevalência }\end{array}$ & $\begin{array}{l}\text { Valor } \\
\text { de } p\end{array}$ & PT+ & PT- & $\begin{array}{l}\text { Razão de } \\
\text { prevalência }\end{array}$ & $\begin{array}{l}\text { Valor } \\
\text { de } p\end{array}$ \\
\hline & $\mathrm{n}(\%)$ & n (\%) & & & $\mathrm{n}(\%)$ & $\mathrm{n}(\%)$ & & \\
\hline \multicolumn{9}{|l|}{ Trabalha em unidade de saúde com PCT } \\
\hline \multicolumn{9}{|l|}{ implantado } \\
\hline Sim & $79(42,10)$ & $109(57,90)$ & & & $118(62,80)$ & $70(37,20)$ & & \\
\hline Não & $36(32,20)$ & $76(67,80)$ & 8,11 & 0,10 * & $58(51,80)$ & $54(48,20)$ & 1,38 & 0,10 * \\
\hline Não sabe & $3(27.20)$ & $8(72,70)$ & & & $6(54,50)$ & $5(45,50)$ & & \\
\hline $\begin{array}{l}\text { Média de tempo que trabalha na função na } \\
\text { atenção básica (anos) }\end{array}$ & 10,20 & 8,40 & 0,63 & $0,00 * \star$ & 9,80 & 8,10 & 1,38 & 0,00 ** \\
\hline \multicolumn{9}{|l|}{ Já trabalhou em ambiente com alto risco de TB? } \\
\hline Sim & $19(32,70)$ & $39(67,30)$ & 0,36 & 0,10 * & $28(48,30)$ & $30(51,70)$ & 1,19 & 0,10 * \\
\hline Não & $95(38,20)$ & $154(61,80)$ & & & $149(59,80)$ & $100(40,20)$ & & \\
\hline \multicolumn{9}{|c|}{ Frequência de EPI disponível na unidade de saúde } \\
\hline Nunca & $7(43,70)$ & $9(56,30)$ & & & $10(62,50)$ & $6(37,50)$ & & \\
\hline Às vezes & $78(36,60)$ & $135(63,40)$ & 5,71 & 0,70 & $122(57,30)$ & $91(42,70)$ & 2,51 & 0,80 \\
\hline Sempre & $32(39,50)$ & $49(60,50)$ & & & $48(59,30)$ & $33(40,70)$ & & \\
\hline \multicolumn{9}{|l|}{ EPI de fácil acesso na unidade de saúde } \\
\hline Sim & $8(16,70)$ & $40(83,30)$ & 3,63 & 0,04 & $25(52,10)$ & $23(47,90)$ & 0,49 & 0,80 \\
\hline Não & $22(33,40)$ & $44(66,60)$ & & & $33(50,00)$ & $33(50,00)$ & & \\
\hline \multicolumn{9}{|c|}{$\begin{array}{l}\text { Frequência de uso do EPI durante atendimento de } \\
\text { paciente sintomático respiratório }\end{array}$} \\
\hline Nunca & $108(36,50)$ & $188(63,50)$ & & & $171(57,70)$ & $125(42,30)$ & & \\
\hline Às vezes & $8(61,50)$ & $5(38,50)$ & 0,04 & 0,10 & $10(76,90)$ & $3(23,10)$ & 5,42 & 0,20 \\
\hline Sempre & $1(33,30)$ & $2(66,70)$ & & & $1(33,30)$ & $2(66,70)$ & & \\
\hline \multicolumn{9}{|l|}{ Recebeu algum treinamento/capacitação sobre } \\
\hline \multicolumn{9}{|l|}{ TB? } \\
\hline No último ano & $51(38,60)$ & $81(61,40)$ & & & $76(57,60)$ & $56(42,40)$ & & \\
\hline $1-2$ anos & $30(32,60)$ & $62(67,00)$ & & & $50(54,40)$ & $42(45,60)$ & & \\
\hline 3-4 anos & $14(41,20)$ & $20(58,80)$ & 8,05 & 0,60 & $22(64,00)$ & $12(35,30)$ & 1,46 & 0,30 \\
\hline 5 anos ou mais & $12(46,20)$ & $14(53,80)$ & & & $19(63,90)$ & $7(26,10)$ & & \\
\hline Não fez & $10(33,30)$ & $20(66,70)$ & & & $15(50,00)$ & $15(50,00)$ & & \\
\hline \multicolumn{9}{|c|}{$\begin{array}{l}\text { Como avalia a contribuição deste treinamento para } \\
\text { prática clínica? }\end{array}$} \\
\hline Boa & $99(38,10)$ & $161(61,90)$ & & 0,40 & $153(58,80)$ & $107(41,20)$ & & \\
\hline Regular & $5(26,30)$ & $14(73,30)$ & 0.33 & & $10(52,70)$ & $9(47,40)$ & 1,74 & 0,60 \\
\hline Ruim & $2(50.00)$ & $2(50,00)$ & & & $1(25,50)$ & $3(75,00)$ & & \\
\hline \multicolumn{9}{|c|}{ Frequência com que busca informações sobre TB } \\
\hline Nunca & $11(25,60)$ & $32(74,40)$ & & & $20(46,50)$ & $23(53,50)$ & & \\
\hline Às vezes & $49(38,30)$ & $79(61,70)$ & 0,04 & 0,20 & $53(41,40)$ & $75(58,60)$ & 1,50 & 0,20 \\
\hline Sempre & $57(39,60)$ & $87(60,40)$ & & & $57(39,60)$ & $87(60,40)$ & & \\
\hline
\end{tabular}

EPI: equipamento de proteção individual; PCT: Programa de Controle da Tuberculose; PT: prova tuberculínica; TB: tuberculose.

Nota: ponto de corte ao resultado da PT - PT+ ( $\geq 10 \mathrm{~mm}$ e $\geq 5 \mathrm{~mm}$ de enduração) e PT- (< $10 \mathrm{~mm}$ e $<5 \mathrm{~mm}$ de enduração).

* Teste de qui-quadrado de Pearson;

** Teste t Student. 
Tabela 3

Fatores associados com os resultados positivos à prova tuberculínica (PT) na análise multivariada - método backward stepwise. Brasil, 2013.

\begin{tabular}{|c|c|c|c|}
\hline Ponto de corte & Variáveis & OR ajustado (IC95\%) & Valor de $p$ \\
\hline \multirow[t]{4}{*}{$\geq 10 \mathrm{~mm}$} & Trabalha em unidade de saúde com PCT implantado & $1,9(1,1-3,4)$ & 0,01 \\
\hline & Já conviveu com alguém com TB & $1,7(1,1-3,0)$ & 0,02 \\
\hline & Formação & & \\
\hline & Ensino universitário & $3,6(1,1-11,9)$ & 0,02 \\
\hline \multirow[t]{3}{*}{$\geq 5 \mathrm{~mm}$} & Trabalha em unidade de saúde com PCT implantado & $4,7(1,7-13,6)$ & 0,00 \\
\hline & Já recebeu algum treinamento sobre TB & & \\
\hline & $1-2$ anos & $0,2(0,07-0,6)$ & 0,00 \\
\hline
\end{tabular}

IC95\%: intervalo de confiança; OR: odds ratio; PCT: Programa de Comtrole da Tuberculose; TB: tuberculose.

a PT. Nesse caso, em países em desenvolvimento, como o Brasil, em virtude da elevada prevalência de infecção tuberculosa na população em geral e do uso da vacina BCG, os resultados podem sofrer interferências 30,31,32.

Nesta pesquisa, os pontos favoráveis foram os seguintes: a realização de um estudo-piloto antes do período da coleta de dados; a padronização na aplicação e leitura da PT e na identificação da cicatriz vacinal de BCG; a disponibilidade de informações sobre o status de HIV obtidas mediante o resultado do teste rápido aplicado a todos os participantes.

Por outro lado, a pesquisa apresenta, também, algumas limitações. Primeiro, o estudo transversal é limitado em sua capacidade de identificar associações causais, de modo que um estudo longitudinal seria mais adequado para identificar as taxas de conversão à PT e os fatores associados. Portanto, um nexo de causalidade entre a prevalência positiva da PT e a transmissão de $M$. tuberculosis não pode ser feita; além disso, as mudanças ao longo do tempo não podem ser monitoradas 13,33. Em segundo lugar, estudos têm avaliado o efeito booster, que variou de 5,8\% a $7,8 \% 34,35$. No presente estudo, esse efeito não foi avaliado, em concordância com a Sociedade Brasileira de Pneumologia e Tisiologia, que, na III Diretrizes para TB, descreve não ser necessária a testagem do efeito booster quando são avaliados os profissionais da saúde 23 .

Outra importante limitação desta investigação é a atual falta de PPD Rt 23 no Brasil e em vários países do mundo, neste período, por falta de matéria-prima. Conforme informado no Oficio Circular 25/CGPNCT/DEVIT/SVS/MS 36, divulgado pelo Ministério da Saúde, não há previsão para abastecimento da rede com PPD. Com isso, o diagnóstico de infecção latente foi muito pre- judicado, pois não há um substituto do teste nas redes de atenção à saúde no Brasil, diferente do que ocorre em outros países, como Canadá e Estados Unidos, por exemplo, onde já está normatizado $37,38,39$. Todavia, sabemos que a principal desvantagem da PT é ter baixa especificidade devido à vacinação prévia com BCG e à exposição a micobactérias não tuberculosas; por conseguinte, a PT superestima a população em risco.

Recentemente, dois novos testes comerciais, baseados na expressão de interferon-gama (IFN- $\gamma$ ) - IGRA -, sintetizados por linfócitos em resposta a antígenos específicos do $M$. tuberculosis (ESAT-6 e CFP-10), vêm se mostrando mais específicos e com maior sensibilidade que a PT na identificação da infecção latente pelo $M$. $t u$ berculosis e TB ativa 37 . Diante do exposto, faz-se necessária a realização de estudos prospectivos em profissionais de saúde, utilizando os IGRA, avaliando a sua aplicabilidade e a relação custoefetividade para a triagem de profissionais da saúde em países com alta prevalência de TB e $100 \%$ de cobertura da vacina BCG.

No presente estudo, foi observada uma predominância de positividade à PT entre os ACS que fazem atendimento direto ao paciente. $\mathrm{O}$ papel do cuidado à saúde deve incluir, além do diagnóstico e do tratamento da doença, a percepção da necessidade de composição de um local com características ambientais favoráveis e comportamentos adequados, cujos membros tenham consciência dos riscos de contraírem a infecção e de suas responsabilidades na prevenção desta. A elaboração de rotinas seguras de trabalho e a orientação dos profissionais minimizam os possíveis riscos 40

Ao analisarmos o conhecimento sobre as ações desenvolvidas pelos ACS no controle da TB e a percepção que estes possuem sobre o 
seu nível de conhecimento e de sua importância no enfrentamento da doença, há deficiência em questões importantes, fundamentais na execução de seu trabalho. Um exemplo é a dificuldade na identificação de casos suspeitos de TB, apontando a necessidade de investigações do processo de formação do conhecimento acerca da enfermidade 41 . A fim de abordar o tema especificamente para os agentes, o Ministério da Saúde, em parceria com a Secretaria de Políticas de Saúde, elaborou uma cartilha 42 com o objetivo de orientá-los sobre o tema e mostrar-lhes algumas medidas de controle da doença. A criação da cartilha e a apropriação dos conteúdos nela contidos poderiam funcionar como uma solução para sanar as dúvidas e auxiliar os agentes no seu trabalho de controle da doença. Entretanto, a cartilha mostra-se insuficiente em seu conteúdo informativo, uma vez que não contém assuntos que interferem diretamente no trabalho dos ACS, tais como efeitos colaterais dos medicamentos, grupos de risco, abordagem de populações de extrema vulnerabilidade social, entre outros.

Inúmeros estudos avaliaram o nível de exposição ocupacional à TB e a positividade à PT $43,44,45,46$. Aqueles que tiveram contato direto e indireto com pacientes com TB foram mais positivos à $\mathrm{PT}{ }^{8}$, concluindo que os que assim trabalham têm o risco aumentado, significativamente associado à exposição.

A obtenção de informações sobre biossegurança foi um diferencial neste estudo. Supõe-se que a indisponibilidade de EPI, conforme apontada pelos ACS, deva-se não somente ao fato de os gestores de saúde dos municípios não atentarem para o potencial risco de transmissão da doença, não requisitando essa medida de proteção respiratória individual, mas também à falta de interesse dos próprios ACS em buscar informações sobre a disponibilidade do equipamento 16,47,48. Embora a máscara N95 seja reconhecidamente um tipo de proteção individual, seu uso não é praticado pela grande maioria, fato corroborado por uma pesquisa com estudantes de enfermagem e medicina da UFES, onde já se observa o incentivo a tal prática desde a formação acadêmica. Contudo, não se observa mudança ou melhora nesse quesito, quando comparado com o estudo conduzido nessa mesma instituição há quase dez anos 16,48. Os ACS relatam ter dificuldades de identificar que medidas de proteção devem usar em suas atividades profissionais, por isso, muitas vezes, atuam de acordo com seu entendimento (valores, crenças, hábitos e costumes) ou conhecimento empírico 49,50.

Outra situação que deve ser considerada, mas que não tem sido muito valorizada na organização e nas avaliações sobre o processo de trabalho desses profissionais, é a sua convivência e proximidade com o usuário portador de TB. Os ACS fazem parte tanto da mesma comunidade de origem de muitos dos quais eles cuidam, quanto do serviço de saúde que assiste o portador de TB. Esse fator leva os agentes a negligenciarem ou mesmo ignorarem, por diversas razões, as medidas de proteção que deveriam adotar na relação institucional com o indivíduo portador da doença. Portanto, há que se refletir se a "intimidade", o conhecimento e a convivência com a clientela, associados aos limites da informação e mesmo de conhecimento, podem se constituir em fatores de desproteção, negligência, constrangimento e limitação, por parte dos ACS, para incorporarem, em sua rotina profissional, as medidas adequadas de proteção no seu agir cotidiano 50 .

Atualmente, a PT não faz parte dos exames admissionais dos agentes comunitários de saúde. Logo, com base nos resultados do presente estudo, sugere-se que a PT seja incluída na rotina de exames admissionais, assim como nos exames periódicos para aqueles profissionais que não são reatores. Como o teste realizado nesta investigação é uma das ferramentas utilizadas para avaliar os cuidados relativos à biossegurança dos trabalhadores, entende-se a necessidade de conscientização nos municípios e o fortalecimento das ações de educação permanente sobre a temática, contemplando os profissionais de saúde, para aumentar a adesão à realização da PT.

Este estudo contribuiu para a elaboração do Plano Nacional de Controle de Infecção de Tuberculose, previsto pela OMS 37 . Uma alta prevalência de ILTB foi observada entre os ACS que trabalham em ambiente de alto risco, em municípios com alta e média prevalência de tuberculose, no Brasil. O conhecimento e a política sobre a doença, assim como a prática pessoal de controle de infecção, devem ser melhorados na atenção básica. Diretrizes abrangentes devem ser desenvolvidas para garantir a saúde dos ACS e reduzir a transmissão da $\mathrm{TB}$ em instituições de saúde. 


\section{Resumen}

Este artículo tiene como objetivo determinar la prevalencia y los factores asociados con la infección por Mycobacterium tuberculosis latente entre los agentes comunitarios de salud (ACS), utilizando dos puntos de corte de la tuberculina: prueba de $5 \mathrm{~mm}$ y $10 \mathrm{~mm}$. Un estudio transversal con los datos recogidos sobre: género, edad, cicatriz de BCG (marca de la vacuna), trabajar en una unidade básica de salud (UBS), vivir en un hogar con tuberculosis, el consumo de alcohol, prueba de la tuberculina (PT) anterior, tabaquismo, antigüedad en la profesión como agente comunitario de salud y comorbilidades. Para controlar las variables de confusión y estimar medidas de efecto (OR) se utilizó la regresión logística. Se aplicó el PT, con una lectura después de 48-72 horas. Las tasas de prevalencia fueron un 57,88\% y 37,3\%, respectivamente, para 5 y $10 \mathrm{~mm}$. Se mantuvo asociado con positividad en el trabajo de corte de $10 \mathrm{~mm}$ en UBS, implementado por el Programa de Control de Tuberculosis (PCT), y que tuvo contacto con la tuberculosis en el hogar. En cuanto al trabajo de $5 \mathrm{~mm}$ de corte en la atención primaria se implementó con el PCT. Se necesitan acciones de sensibilización en los municipios.

Prueba de Tuberculina; Tuberculosis; Mycobacterium tuberculosis; Agentes Comunitarios de Salud

\section{Colaboradores}

E. L. N. Maciel, W. P. Rogerio e R. C. D. Lima contribuíram na concepção e projeto do artigo, análise e interpretação dos dados, redação do texto, revisão crítica relevante do conteúdo intelectual e aprovação final da versão a ser publicada. T. N. Prado colaborou na concepção e projeto do artigo, participou da coleta de dados, colaborou na análise estatística dos dados, na elaboração e discussão dos resultados e redação do texto; revisão crítica relevante do conteúdo intelectual e aprovação final da versão a ser publicada. F. M. Souza participou da coleta de dados, colaborou na análise estatística dos dados, na elaboração e discussão dos resultados e redação do texto. J. S. Pinheiro, P. M. Rodrigues e A. P. N. Sant'anna K. G. Jesus participaram da coleta de dados e de todas as etapas da produção do artigo. C. Cerutti Junior contribuiu na interpretação dos dados; redação do texto; revisão crítica relevante do conteúdo intelectual e aprovação final da versão a ser publicada.

\section{Agradecimentos}

Agradecemos aos integrantes do grupo de pesquisa Laboratório de Epidemiologia da Universidade Federal do Espírito Santo, pelo apoio durante todas as etapas do artigo. Às Secretarias Municipais de Saúde. À FAPES e ao CNPq, pelo financiamento.

\section{Referências}

1. Secretaria de Vigilância em Saúde, Ministério da Saúde. Tuberculose: alinhada com o social, afinada com a tecnologia. Boletim Epidemiológico 2013; 44(2).

2. World Health Organization. Global tuberculosis control: surveillance, planning, financing. Geneva: World Health Organization; 2007.

3. Ministério da Saúde. Manual de normas para o controle da tuberculose. 4a Ed. Brasília: Ministério da Saúde; 2002.

4. World Health Organization. Global tuberculosis report 2013. Geneva: World Health Organization; 2013.
5. Maciel EL, Bussinguer ECA, Andrade MAC, Lima RCD. Territorialização em saúde e liberdade individual: desafios à consolidação do SUS. In: Atanaka-Santos M, Pignatti MG, Chaves AL, organizadores. Questões ambientais em saúde coletiva. Cuiabá: EdUFMT; 2012. p. 39-56.

6. Muniz JN, Villa TC, Pedersolli CE. Tratamento supervisionado no controle da tuberculose em Ribeirão Preto: novo modo de agir em saúde. Bol Pneumol Sanit 1999; 7:33-42. 
7. Nunes MO, Trad LB, Almeida BA, Homem CR, Melo MCIC. O agente comunitário de saúde: construção da identidade desse personagem híbrido e polifônico. Cad Saúde Pública 2002; 18:1639-46.

8. Moreira TR, Zandonade E, Maciel ELN. Risco de infecção tuberculosa em agentes comunitários de saúde. Rev Saúde Pública 2010; 44:332-8.

9. Rodrigues PM, Moreira TR, Moraes AKL, Vieira RCA, Dietze R, Lima RCD, et al. Infecção por Mycobacterium tuberculosis entre agentes comunitários de saúde que atuam no controle da TB. J Bras Pneumol 2009; 35:351-8.

10. Oliveira JS, Possuelo LG, Severo K, Carneiro M, Krummenauer E, Machado CP, et al. Avaliação da positividade ao teste tuberculínico entre os trabalhadores da rede básica de saúde. Rev HCPA \& Fac Med Univ Fed Rio Gd do Sul 2011; 31:13-7.

11. World Health Organization. Implementing the Stop TB Strategy: a handbook for national tuberculosis control programmes. Geneva: World Health Organization; 2008.

12. Minayo MC, D’Elia JC, Suitone E. Programa Agente Comunitário de Saúde do Ceará: estudo de caso. Fortaleza: Fundo das Nações Unidas para a Infância; 1990.

13. Silva VM, Cunha AJ, Kritski AL. Risco de infecção pelo Mycobacterium tuberculosis entre alunos da Faculdade de Medicina da Universidade Federal do Rio de Janeiro. J Bras Pneumol 2004; 30:459-66.

14. Silva JA. O agente comunitário de saúde do projeto Qualis: agente institucional ou agente da comunidade? [Tese de Doutorado]. São Paulo: Faculdade de Saúde Pública, Universidade de São Paulo; 2001.

15. Franco C, Zanetta DM. Tuberculose em profissionais de saúde: medidas institucionais de prevenção e controle. Arq Ciênc Saúde 2004; 11:244-52.

16. Maciel EL, Viana MC, Zeitoune RC, Ferreira I, Fregona G, Dietze R. Prevalence and incidence of Mycobacterium tuberculosis infection in nursing students in Vitória, Espírito Santo. Rev Soc Bras Med Trop 2005; 38:469-72.

17. Prado TN, Galavote HS, Brioschi AP, Lacerda T, Fregona G, Dettoni VV, et al. Perfil epidemiológico dos casos notificados de tuberculose entre os profissionais de saúde no Hospital Universitário em Vitória-ES, Brasil. J Bras Pneumol 2008; 34:607-13.

18. Maciel EL, Meireles W, Silva AP, Fiorotti K, Dietze R. Nosocomial Mycobacterium tuberculosis transmission among healthcare students in a high in cidence region, in Vitória, State of Espírito Santo. Rev Soc Bras Med Trop 2007; 40:397-9.

19. Oliveira SM, Honner MR, Paniago AM, Aguiar ES, Cunha RV. Prevalência da infecção tuberculosa entre profissionais de um hospital universitário. Rev Latinoam Enferm 2007; 15:1120-4.

20. Maciel EL, Prado TN, Fávero JL, Moreira TR, Dietze R. Tuberculose em profissionais de saúde: um novo olhar sobre um antigo problema. J Bras Pneumol 2009; 35:83-90.

21. Joshi R, Reingold AL, Menzies D, Pai M. Tuberculosis among health-care workers in low- and middleincome countries: a systematic review. PLoS Med 2006; 3:e494.
22. Roth VR, Garrett DO, Laserson KF, Starling CE, Kritski AL, Medeiros EA, et al. A multicenter evaluation of tuberculin skin test positivity and conversion among health care workers in Brazilian hospitals. Int J Tuberc Lung Dis 2005; 9:1335-42.

23. Departamento de Vigilância Epidemiológica, Secretaria de Vigilância em Saúde, Ministério da Saúde. Manual de recomendações para o controle da tuberculose no Brasil. Brasília: Ministério da Saúde; 2011.

24. Cailleaux CM, Melo DA, Xavier GM, Salles CL, Mello FC, Ruffino NA, et al. Tuberculosis incidence among contacts of active pulmonary tuberculosis. Int J Tuberc Lung Dis 2009; 13:190-5.

25. Oliveira GP, Torrens AW, Bartholomay P, Barreira D. Tuberculosis in Brazil: last ten years analysis 2001-2010. Braz J Infect Dis 2013; 17:218-33.

26. Barros AJ, Hirakata UM. Alternatives for logistic regression in cross-sectional studies: an empirical comparison of models that directly estimate the prevalence ratio. BMC Med Res Methodol 2003; 3:21.

27. Conde MB, Melo FAF, Marques AMC, Cardoso NC, Pinheiro VGF, Dalcin PTR, et al. III diretrizes brasileiras para tuberculose da Sociedade Brasileira de Pneumologia e Tisiologia. J Bras Pneumol 2009; 35:1018-48.

28. Menzies D. Interpretation of repeated tuberculin tests. Boosting, conversion, and reversion. Am J Respir Crit Care Med 1999; 159:15-21.

29. Moreno S, Blázquez R, Novoa A, Carpena I, Menasalvas A, Ramírez C, et al. The effect of BCG vaccination on tuberculin reactivity and the booster effect among hospital employees. Arch Intern Med 2001; 161:1760-5.

30. Quadros CM, Biscotto CR, Tinois BR, Freitas FC, Oliveira JS, Azevedo MF, et al. Prevalência da infecção tuberculosa em universitários da Universidade Estadual de Montes Claros, Minas Gerais. Rev Bras Educ Méd 2009; 33:535-41.

31. Ferreira FM. Prevalência de infecção por Mycobacterium tuberculosis em estudantes de medicina da Universidade do Rio de Janeiro [Dissertação de Mestrado]. Rio de Janeiro: Universidade Federal do Rio de Janeiro; 1998.

32. Mazurek GH, LoBue PA, Daley CL, Bernardo J, Lardizabal AA, Bishai WR, et al. Comparison of a whole blood interferon gamma assay with tuberculin skin testing for detecting latent mycobacterium tuberculosis infection. JAMA 2001; 286:1740-7.

33. Silva VMC, Oliveira JR, Santos FM, Araújo CM, Kritski AL. Prevalência de infecção pelo Mycobacterium tuberculosis entre alunos da Faculdade de Medicina da Universidade Federal do Rio de Janeiro. J Bras Pneumol 2001; 27:77-82.

34. Schwartzman K, Loo V, Pasztor J, Menzies D. Tuberculosis infection among health care workers in Montreal. Am J Respir Crit Care Med 1996; 154:1006-12.

35. Pereira AL, Bolzani FC, Stefani M, Charlín R. Uso sistêmico de corticosteróides: revisão da literatura. Med Cutan Iber Lat Am 2007; 35:35-50. 
36. Coordenação Geral do Programa Nacional de Controle da Tuberculose, Secretaria de Vigilância em Saúde, Departamento de Vigilância das Doenças Transmissíveis, Ministério da Saúde. Ofício Circular no 25/CGPNCT/DEVIT/SVS/MS. Dificuldades na aquisição do derivado proteico purificado - PPD. http://www.cve.saude.sp.gov.br/htm/TB/ eventos/TB_OfCircular25_PPD.pdf (acessado em 01/Ago/2014).

37. Pai M, Denkinger CM, Kik SV, Rangaka MX, Zwerling A, Oxlade O. Gamma interferon release assays for detection of Mycobacterium tuberculosis infection. Clin Microbiol Rev 2014; 27:3-20.

38. Canadian Tuberculosis Committee. Recommendations on interferon gamma release assays for the diagnosis of latent tuberculosis infection - 2010 update. Canada Communicable Disease Report 2010; 36:1-22.

39. Mazurek M, Jereb J, Vernon A, LoBue P, Goldberg S, Castro K. Updated guidelines for using interferon gamma release assays to detect Mycobacterium tuberculosis infection - United States, 2010. MMWR Recomm Rep 2010; 59:1-25.

40. Chermont AB, Braga AV, Carvalho CM, Queiroz DM, Gonçalves FJ, Franco FP, et al. Incidência de tuberculose pulmonar em profissionais de saúde. In: XI Congresso Médico de Pneumologia e Tisiologia do Rio de Janeiro. http://www.metodoeven tosrio.com.br/Pneumo2007/chamada.htm (acessado em 20/Jul/2013).

41. Maciel EL, Vieira RC, Milani EC, Brasil M, Fregona G, Dietze R. O agente comunitário de saúde no controle da tuberculose: conhecimentos e percepções. Cad Saúde Pública 2008; 24:1377-86.

42. Ministério da Saúde. Tuberculose, informações para agentes comunitários de saúde. Boletim Alerta $2001 ; 7(4)$.

43. Lien LT, Hang NT, Kobayashi N, Yanai H, Toyota E, Sakurada S, et al. Prevalence and risk factors for tuberculosis infection among hospital workers in Hanoi, Viet Nam. PLoS Med 2009; 4:e6798.
44. Do AN, Limpakarnjarat K, Uthaivoravit W, Zuber PL, Korattana S, Binkin N, et al. Increased of Mycobacterium tuberculosis infection related to the occupational exposures of health care workers in Chiang Rai, Thailand. Int J Tuberc Lung Dis 1999; 3:377-81.

45. Kassim S, Zuber P, Wiktor SZ, Diomande FV, Coulibaly IM, Coulibaly D, et al. Tuberculin skin testing to assess the occupational risk of Mycobacterium tuberculosis infection among health care workers in Abidjan, Côte d'Ívoire. Int J Tuberc Lung Dis 2000; 4:321-6.

46. Larsen NM, Biddle CL, Sotir MJ, White N, Parrott P, Blumberg HM. Risk of tuberculin skin test conversion among health care workers: occupational versus community exposure and infection. Clin Infect Dis 2002; 35:796-801.

47. Rogerio WP, Baraona CM, Prado TN, Lacerda TC Carlesso GF, Maciel EL. Prevalência de infecção latente pelo Mycobacterium tuberculosis entre estudantes da área da saúde de uma universidade pública em Vitória, ES, Brasil. Ciênc Saúde Coletiva 2013; 18:1331-9.

48. Yushiara EV. Conhecimento sobre as medidas de biossegurança para tuberculose nos Agentes Comunitários de Saúde no Município de Vitória, ES [Dissertação de Mestrado]. Vitória: Universidade Federal do Espírito Santo; 2013.

49. Pôrto A. Representações sociais da tuberculose: estigma e preconceito. Rev Saúde Pública 2007; 41 Suppl 1:43-9.

50. World Health Organization. WHO policy on TB infection control in health care facilities, congregate settings and households. Geneva: World Health Organization; 2009.

Recebido em 10/Out/2014

Versão final reapresentada em 22/Mar/2015

Aprovado em 04/Mai/2015 